\title{
Lévy-Brownian motion on finite intervals: Mean first passage time analysis.
}

\author{
B. Dybied and E. Gudowska-Nowakif \\ Marian Smoluchowski Institute of Physics and Mark Kac Center for Complex Systems Research, \\ Jagellonian University, ul. Reymonta 4, 30-059 Kraków, Poland \\ P. Hängg团 \\ Institute of Physics, University of Augsburg, Universitätsstrasse 1, 86135 Augsburg, Germany
}

(Dated: February 2, 2008)

\begin{abstract}
We present the analysis of the first passage time problem on a finite interval for the generalized Wiener process that is driven by Lévy stable noises. The complexity of the first passage time statistics (mean first passage time, cumulative first passage time distribution) is elucidated together with a discussion of the proper setup of corresponding boundary conditions that correctly yield the statistics of first passages for these non-Gaussian noises. The validity of the method is tested numerically and compared against analytical formulae when the stability index $\alpha$ approaches 2, recovering in this limit the standard results for the Fokker-Planck dynamics driven by Gaussian white noise.
\end{abstract}

PACS numbers: 02.50.Ey, 05.10.Gg, 05.10.Ln

\section{INTRODUCTION}

Stochastic Lévy processes serve as paradigms for the description of many unusual transport processes leading to anomalous diffusion as characterized by an anomalous mean squared displacement, i.e.,

$$
\left\langle(x(t)-\langle x(t)\rangle)^{2}\right\rangle=\left\langle(\Delta x)^{2}\right\rangle \propto t^{\nu}
$$

which deviates with $\nu \neq 1$ from the linear dependence $\left\langle(\Delta x)^{2}\right\rangle \propto t$ that characterizes normal diffusion. In the above formula $\nu$ stands for the anomalous diffusion exponent that specifies the process at hand as either behaving subdiffusive (with $0<\nu<1$ ), superdiffusive $(1<\nu)$ or ballistic (for $\nu=2$ ) 1, 2, 3, 4]. Among the class of Lévy processes, the free Lévy flights (LF) represent a special class of discontinuous Markovian processes, for which the mean squared displacement, as defined in equation (11) diverges due to the heavy-tail distribution of the independent increments $\Delta x$. In this case, the mean squared displacement is always superdiffusive; i.e. $\left\langle(x(t)-\langle x(t)\rangle)^{2}\right\rangle \propto t^{2 / \alpha}$, where $0<\alpha<2$ denotes the stability index of the Lévy-Brownian motion process, see below in Sect. II. In contrast to the spatio-temporal coupling characterizing general forms of non-Markovian, or more precisely, semi-Markovian 2 Lévy walks (LW) 1, 3, 4], Lévy flights correspond to the class of Markov processes that emerge from a Langevin equation with $\delta$ correlated, white Lévy noise. Because LFs typically possess a broad jump length distribution with an asymptotic power law behavior their trajectories display at all scales self-similar clustering of local sojourns that become interrupted by long jumps into the other location in the phase

\footnotetext{
*Electronic address: bartek@th.if.uj.edu.pl

${ }^{\dagger}$ Electronic address: gudowska@th.if.uj.edu.pl

${ }^{\ddagger}$ Electronic address: peter.hanggi@physik.uni-augsburg.de
}

space where a new clustering forms. Early discoveries of LF-like phenomena were related to intermittent chaotic systems and description of the motion of the fluid particles in fully developed turbulence [1, 2, 3]. Nowadays, their applications range from description of the dynamics in plasmas, diffusion in the energy space, self-diffusion in micelle systems and transport in polymer systems under conformational motion [1, 5] to the spectral analysis of paleoclimatic [6] or economic data []]. Despite the ubiquitous use of LFs as phenomenological models for noise sources, their influence on the kinetics subjected to boundary conditions has been addressed scarcely only. For free normal diffusion, the knowledge of the Green function, together with the local boundary conditions, is sufficient to determine the first passage time statistics. The same information can be also obtained by use of the method of images or by solving the corresponding, local boundary value problem of the diffusive Fokker-Planck equation [8, 9].

In particular, for processes driven by white Gaussian noise these boundary conditions for reflection or absorption are locally defined and well known [9, 10]. However, for the case of LFs, the method of images fails, yielding results that contradict the Sparre-Andersen theorem [11, 12]. By virtue of the latter [13], for any discrete time random walk starting out at $x_{0} \neq 0$ with the step length sampled from a continuous, symmetric distribution, the first passage time density decays asymptotically as $t^{-3 / 2}$. In order to further explore the intricate problem of proper boundary conditions for absorption and reflection for non-Gaussian white noise composed of independent Lévy flight increments, we elucidate with this work the situation of a free, overdamped Lévy-Brownian motion, being restricted to a bounded domain of attraction. 


\section{RESTRICTED LÉVY-BROWNIAN MOTION}

We consider the free Brownian motion on a restricted, finite interval that is driven by Lévy stable noise. More specifically, the dynamical evolution of a stochastic state variable $x(t)$ is described in terms of the Langevin equation

$$
\frac{d x(t)}{d t}=\zeta(t)
$$

where $\zeta(t)$ denotes a Lévy stable white noise process which is composed of independent differential increments that are distributed according to the stable density with the index $\alpha$; i.e. $L_{\alpha, \beta}(\zeta ; \sigma, \mu=0)$. Put differently, $\zeta(t)$ stands for the generalized white noise process which is obtained from the time derivative of the corresponding Lévy-Brownian (Markovian) stable process. The parameter choice $\alpha=2$ yields the usual, $\delta$-correlated Gaussian white noise. In contrast, with $0<\alpha<2$ the corresponding Lévy white noise is generated from LévyBrownian motion possessing discontinuous sample paths with infinite variance and the higher cumulants. Its statitiscal properties can be however characterized by fractional moments of order $\nu$ which exist and are finte for $\nu<\alpha<2$ [14]. Because it is composed of independent increments, this Lévy stable white noise also constitutes a singular white noise process whose autocorrelation again is formally $\delta$-correlated [14]. Here, the parameter $\alpha$ denotes the stability index, yielding the asymptotic power law for the jump length distribution being proportional to $|\zeta|^{-1-\alpha}$. The parameter $\sigma$ characterizes a scale, $\beta$ defines an asymmetry (skewness) of the distribution, whereas $\mu$ denotes the location parameter. We deal only with strictly stable distributions not exhibiting a drift regime; this implies a vanishing location parameter $\mu=0$ throughout the remaining part of this work. For $\alpha \neq 1$, the characteristic function $\phi(k)=\int_{-\infty}^{\infty} e^{-i k \zeta} L_{\alpha, \beta}(\zeta ; \sigma, \mu=0) d \zeta$ of an $\alpha$-stable random variable $\zeta$ can be represented by

$$
\phi(k)=\exp \left[-\sigma^{\alpha}|k|^{\alpha}\left(1-i \beta \operatorname{sign}(k) \tan \frac{\pi \alpha}{2}\right)\right],
$$

while for $\alpha=1$ this expression reads

$$
\phi(k)=\exp \left[-\sigma|k|\left(1+i \beta \frac{2}{\pi} \operatorname{sign}(k) \ln |k|\right)\right] .
$$

The three remaining parameters vary within the regimes $\alpha \in(0,2], \beta \in[-1,1], \sigma \in(0, \infty)$.

The stochastic differential equation in (2) yields normal, free Brownian motion when $\alpha=2$, and free superdiffusion when $\alpha \in(0,2)$. The numerical integration of equation (2) has been performed by use of standard techniques of integration of stochastic differential equation with respect to the Lévy stable measures and studied by use of the Monte Carlo methods 15]. In particular, the error bars visible in the figures were calculated using the bootstrap method. The position of a LévyBrownian particle has been obtained by a direct integration of Eq. (21) leading to the following approximation 16, 17, 18, 19, 20, 21]

$$
x(t)=\int_{0}^{t} \zeta(s) d s \approx \sum_{i=0}^{N-1}(\Delta s)^{1 / \alpha} \zeta_{i},
$$

where $\zeta_{i}$ are independent random variables distributed with the probability density function (PDF) $L_{\alpha, \beta}(\zeta ; \sigma, \mu=0)$ and $N \Delta s=t$. All our illustrations in this work are based on trajectory calculations sampled from the Langevin equation (2). Absorbing boundary conditions have been realized by stopping the trajectory whenever it reached the boundary, or, more typically, it has jumped beyond that boundary location. The condition of reflection has been assured by wrapping the hitting (or crossing) trajectory around the boundary location, while preserving its assigned length. The Appendix A provides some further details on the numerical scheme for stochastic differential equations driven by Lévy white noise. We also like to emphasize that our results omit cases when $\alpha=1$ with $\beta \neq 0$. In fact, this parameter set is known to induce instabilities in the numerical evaluation of corresponding trajectories [16, 17, 18, 19, 20, 21]. Thus, for our numerics with $\beta \neq 0$, the parameter value $\alpha=1$ has been excluded from the consideration.

\section{MEAN FIRST PASSAGE TIMES FOR $\alpha$-STABLE NOISES}

Our main objective is the investigation of the mean first passage time (MFPT) of Lévy-Brownian motion on a finite interval as illustrated with Fig. 1. The boundaries at both ends will be assumed to be of either absorbing or also of the reflecting type. With generally non-Gaussian white noise the knowledge of the boundary location alone cannot specify in full the corresponding boundary conditions for absorption or reflection, respectively. In particular, the trajectories driven by non-Gaussian white noise depict discontinuous jumps, cf. Figs. 2 and 4 below. As a consequence, the location of the boundary itself is not hit by the majority of discontinuous sample trajectories. This implies that regimes beyond the location of the boundaries must be properly accounted for when setting up the boundary conditions. Most importantly, returns (i.e. so termed re-crossings of the boundary location) from excursions beyond the specified state space back into this very finite interval where the process proceeds must be excluded. Thus, the problem of proper formulation of boundary conditions in such cases poses an open challenge that has not been addressed with sufficient care in the prior literature 22, 24]. In contrast to the case with normal diffusion (i.e. when $\alpha=2, \beta=0$ ), these boundary conditions are of a nonlocal nature; as a consequence, an analytical investigation of the mean first passage problem becomes very demanding and cumbersome. In this work, we therefore restrict ourselves 
predominantly (for $\alpha<2$ ) to detailed, precise numerical simulations.

The setup for the studies is schematically depicted in Fig. 11 The stochastic motion of a free Lévy particle is confined in an interval specified by the two boundaries $B_{1}, B_{2}$. The dynamics of the LFs trajectories derived from Eq. (2) are consequently confined to this state space in-between $B_{1}$ and $B_{2}$.

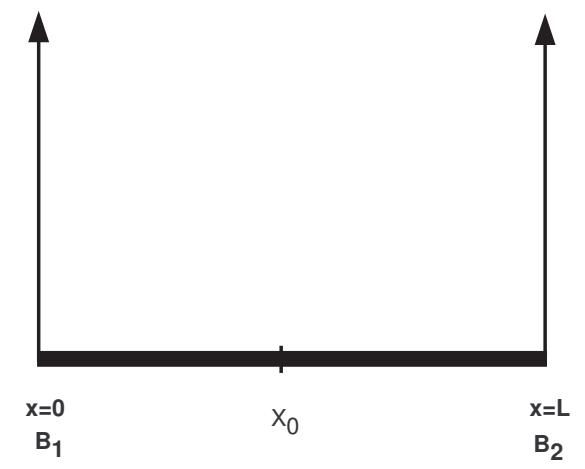

FIG. 1: Setup for the investigation of the first passage time analysis of Lévy white noise driven free Brownian motion being confined between the two boundaries located at $B_{1}$ and $B_{2}$, respectively.
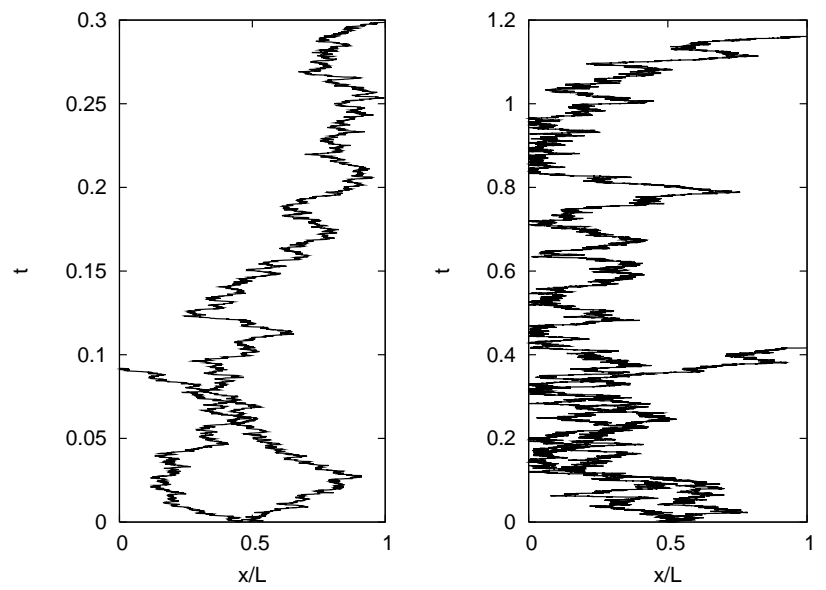

FIG. 2: Left panel: Typical sample trajectories of confined, normal diffusion, i.e. $\alpha=2$, with two absorbing AA boundaries. Right panel: Confined normal Brownian motion between a left-sided reflecting boundary $(\mathrm{R})$ and a right-sided absorbing boundary (A), denoted as RA in the text. The particles start out at midpoint $x / L=0.5$, undergoing statecontinuous stochastic motion with evolving time $t$.

\section{A. The test case: normal diffusion}

For $\alpha=2$ (see Fig. 2), white Lévy stable noise becomes equivalent to Gaussian white noise and the corresponding Langevin eqution (2) describes a free Brownian motion
(Wiener process) for which the probability density functions (PDFs) of the first passage times distributions are known explicitly from the literature [8, 9]. Here, due to reasons of convenience, instead of estimating the PDFs itself we simulate the equivalent cumulative distribution functions (CDFs) of first passage times. For the sake of simplicity it has been assumed that the left boundary is located at $x=0$ and the other one at $x=L$. In the following we typically use, although not exclusively, for the initial condition, $x_{0}$, the center of the interval, $x_{0}=L / 2$. In the case when both $B_{1}$ and $B_{2}$ are absorbing boundaries, denoted as AA, the cumulative distribution function $\mathcal{F}$ of first passage times has been obtained by integration of the first passage time density $f(t)$, i.e. $\mathcal{F}(t)=\int_{0}^{t} f\left(t^{\prime}\right) d t^{\prime}[\underline{8},[9,10]$ :

$\mathcal{F}(t)=1-\frac{2}{\pi} \sum_{j=1}^{\infty} \frac{1-\cos j \pi}{j} \sin \frac{j \pi x_{0}}{L} \exp \left[-\left(\frac{j \pi \sigma}{L}\right)^{2} t\right]$.

Analogously, for a left absorbing boundary (A) and a reflecting right boundary $(\mathrm{R})$, denoted as $\mathrm{AR}$, the corresponding $\mathrm{CDF}$ of first passage times reads [8, 9, 10]

$$
\begin{aligned}
\mathcal{F}(t) & =\frac{4}{\pi} \sum_{j=0}^{\infty} \frac{1}{2 j+1} \sin \frac{(2 j+1) \pi}{2} \cos \frac{(2 j+1) \pi\left(L-x_{0}\right)}{2 L} \\
& \times\left[1-\exp \left[-\left(\frac{(2 j+1) \sigma \pi}{2 L}\right)^{2} t\right]\right]
\end{aligned}
$$

Finally, for a left reflecting boundary $(\mathrm{R})$ and a absorbing right boundary $(\mathrm{A})$, denoted as RA, the corresponding $\mathrm{CDF}$ of first passage times reads [8, 9, 10]

$$
\begin{aligned}
\mathcal{F}(t) & =\frac{4}{\pi} \sum_{j=0}^{\infty} \frac{1}{2 j+1} \sin \frac{(2 j+1) \pi}{2} \cos \frac{(2 j+1) \pi x_{0}}{2 L} \\
& \times\left[1-\exp \left[-\left(\frac{(2 j+1) \sigma \pi}{2 L}\right)^{2} t\right]\right] .
\end{aligned}
$$

where the scaling parameter $\sigma$ stands for the amplitude of the noise intensity for the additive white Gaussian noise; i.e. $\langle\zeta(t) \zeta(s)\rangle=2 \sigma^{2} \delta(t-s)$. Note, that for a particle starting its motion at $x_{0}=L / 2, \mathcal{F}(t)$ for a RA boundary setup is the same as for the symmetrically chosen AR situation, cf. the formulae above. To test our employed software, we evaluated numerically the first passage times distributions with the same boundary conditions as specified above. Eq. (2) has been integrated numerically for $B_{1}=0, B_{2}=5, x_{0}=3.5$ with $\Delta t=10^{-5}$ and averaged over $N=3 \times 10^{4}$ realizations. Figure 3 depicts the numerical results along with their corresponding analytical expressions for the corresponding compound distribution functions (CDFs) of first passage times for a Lévy-Gaussian case $(\alpha=2)$. We find a perfect agreement between the theoretical and the numerical results. On purpose, we have chosen here an asymmetric starting point in order to impose an explicit difference between the AR and the corresponding RA setup, respectively. 


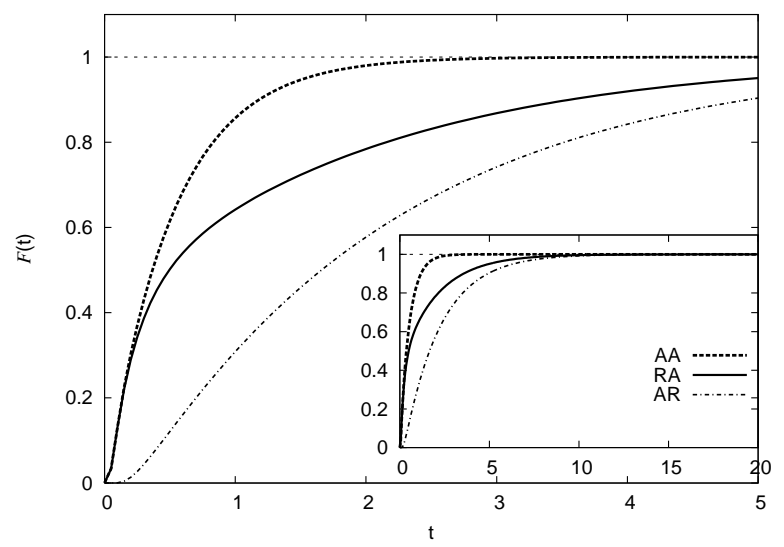

FIG. 3: First passage time statistics for free normal diffusion $(\alpha=2)$ occurring between two boundaries located at $x=0$ and $x=5$. The cumulative first passage time distribution $\mathcal{F}(t)$ is depicted versus the first passage time variable $t$. The initial motion starts out at $x_{0}=3.5$ and the noise strength is $\sigma^{2}=5$. The chosen time step is $\Delta t=10^{-5}$ and the results have been averaged over $N=3 \times 10^{4}$ realizations. The simulation results are presented for AA, RA and AR configurations (from the top to the bottom) along with lines representing the analytical formulae for these cases. The numerical data match perfectly the analytical results, plotted on superimposed lines. The inset depicts this agreement on an extended time span up to time $t=20$.

\section{B. Confined Lévy-Brownian motion}

After having tested the numerical algorithm, we next study the mean first passage time for confined LévyBrownian motion on a finite interval.

\section{MFPT for symmetric Lévy noise}

Using the discussed simulation procedure we start out with the case of confined Lévy-Brownian motion with symmetric stable Lévy noise, i.e. we set $\beta=0$. Exem-

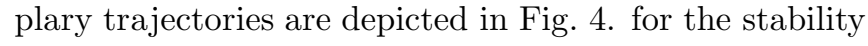
index $\alpha=1.4$. In clear contrast to normal Brownian motion we detect discontinuous jumps, characterizing random jumps over large distances (note the horizontal excursions in Fig. (4). As a consequence, the boundaries at $B_{1}, B_{2}$ are typically not hit, but rather crossed in a flight-like manner, being characteristic for Lévy distributed jumps. In fact, for $\alpha<2$ large excursions of the trajectory are more probable than for normal Brownian motion. In effect, a test "particle" can skip over the border, i.e. it can escape from the domain of motion via a single jump.

As discussed in the literature [1, 3, 11], the scaling nature of the jump length PDFs causes a clustering of Lévy flights. Random localized motion is occasionally interrupted by long sojourns on all length scales and, additionally, there are clusters of local motion within clus-
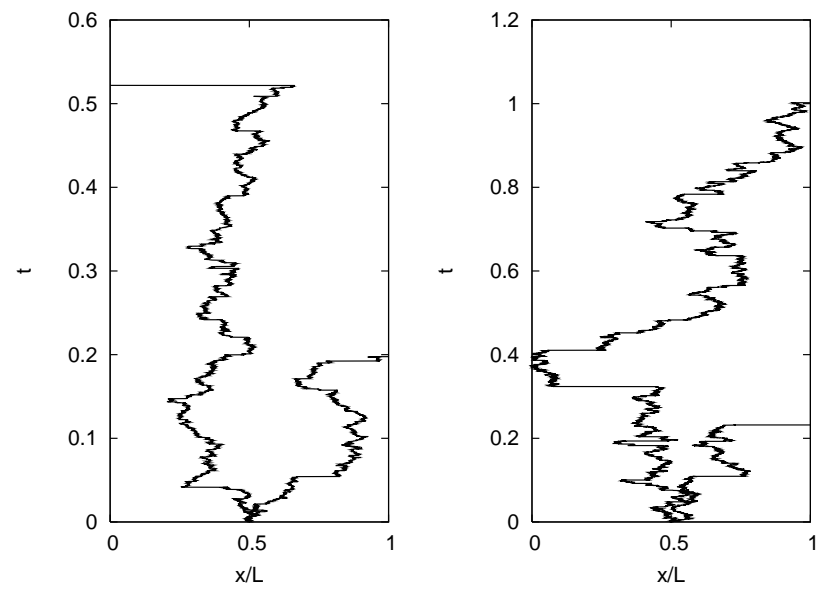

FIG. 4: Sample trajectories for AA (left panel) and RA (right panel) with the two boundaries located at $B_{1}=0$ and $B_{2}=L$ for symmetric Lévy flights with $\alpha=1.4, \beta=0$. For $\alpha<2$ large excursions of the trajectory are more probable than for the normal Brownian motion. A test "particle" can skip over the border, i.e. it can escape from the domain of motion with a single jump.

ters. Anomalous trajectories of Lévy flights with stability index $\alpha<2$ influence also the boundary condition for the mean first passage time and its corresponding statistics.

From the perspective of a random walk approximation to the Langevin equation in (2), the behavior of a Lévy walker is drastically different for Lévy jump length statistics as compared with a traditional Gaussian case: The increments of the normal (state-continuous) diffusion process $x(t)$ are characterized by statistics which excludes with very large probability the occurrence of long jumps. Therefore, the walker is more probable to approach - and eventually hit - a point-like boundary (cf. Fig. (2). In contrast (see Fig. (4), with the Lévy jump statistics $(\alpha<2)$, a meandering particle may easily cross the local boundary during its long jump and may recross into the finite interval many times, unless the particle is immediately absorbed upon crossing for the first time the boundary $B_{1}$ or $B_{2}$, respectively. This brings about a formulation of the boundary condition that necessarily must be non-local in nature.

Indeed, the first passage time problem for Eq. (2) can also be rephrased in terms of the fractional Fokker-Planck equation [11, 25, 26, 27, 28, 29]. For a free LévyBrownian motion this equation with $\beta=0$ assumes the form:

$$
\frac{\partial p(x, t)}{\partial t}=\sigma^{\alpha} \frac{\partial^{\alpha}}{\partial|x|^{\alpha}} p(x, t) .
$$

The boundary conditions for the first passage time problem associated with two absorbing boundaries at $B_{1}$ and $B_{2}$ are now nonlocal; reading,

$$
p(x, t)=0 \text { for } x \leqslant B_{1} \text { and } p(x, t)=0 \text { for } x \geqslant B_{2} .
$$

Due to discontinuous character of trajectories of Lévy 
processes we note that the usual form of boundary conditions, i.e. $p(x=0, t)=0$ and $p(x=L, t)=0$, incorrectly employed in the literature [22, 24, 30, 31] is expected to lead to erroneous results; such a boundary condition does not account for the fact that the process can skip the location of the boundary without hitting it. The corresponding MFPT can be numerically integrated, yielding

$$
M F P T=\int_{0}^{\infty}-t d t \int_{B_{1}}^{B_{2}} \dot{p}(x, t) d x
$$

which after a partial integration equals

$$
M F P T=\int_{0}^{\infty} d t \int_{B_{1}}^{B_{2}} p(x, t) d x .
$$

This expression has been tested versus the statistical definition based on histogram analysis of the corresponding trajectories Eq. (2), for which the appropriate boundary conditions were imposed.

Likewise, we also have studied the case where one of the two boundaries becomes reflecting. The reflecting boundary is imposed on the trajectory simulations via an infinite high hard wall, yielding immediate reflection 32]. For the numerical implementation of this reflecting case see below Eq. [5] In Fig. [5 we compare our numerical results for the case of two absorbing boundaries, case AA, and as well, for the symmetric situation, $\mathrm{AR}=\mathrm{RA}$, of a reflecting boundary and an absorbing boundary with the initial starting value chosen at midpoint.
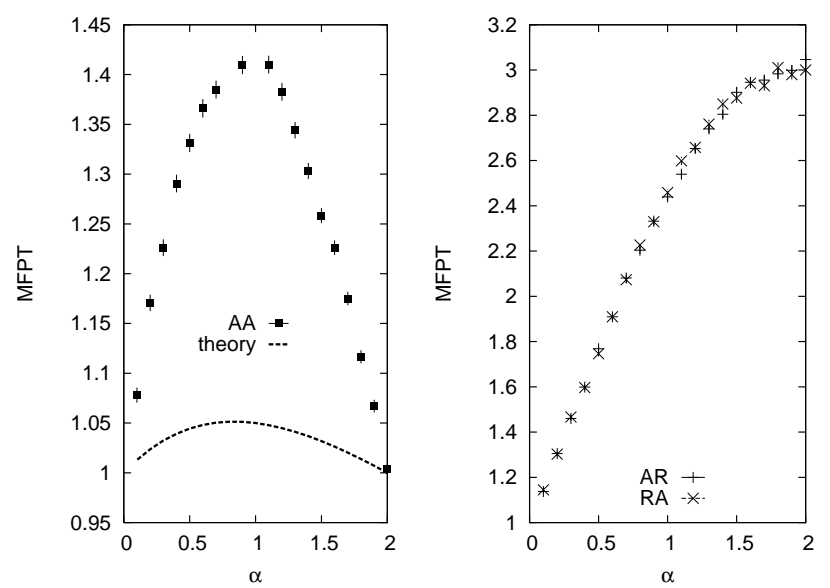

FIG. 5: Mean first passage time versus the stability index $\alpha$ of confined Brownian motion driven by stable symmetric Lévy noise. We depict the case of two absorbing boundaries, AA (left panel), and the situation of a reflecting and absorbing barrier, AR/RA (right panel). The initial starting point has been chosen at midpoint $x_{0}=2$, yielding identical results for $\mathrm{AR}$ and $\mathrm{RA}$, respectively. The simulations parameters are: $\beta=0, \sigma=\sqrt{2}$, time step $\Delta t=10^{-4}$, number of realizations $N=2 \times 10^{2}$. The boundaries are located at $B_{1}=0$ and $B_{2}=4$. The theory-result from Ref. [22] (Eq. (39) therein) is plotted as a dashed line.
Fig. [5 depicts the numerical results for the MFPT for symmetric Lévy stable noise after implementing numerically the appropriate boundary conditions as discussed above. In the left panel of Fig. 5 the numerical results for the MFPT are compared with the theoretical findings for the MFPT $\equiv T$ in this superdiffusive case, reading from Eq. (39) in Refs. 22, 23],

$$
T=\frac{4}{\pi D_{\alpha}}\left(\frac{L}{\pi}\right)^{\alpha} \sum_{m=0}^{\infty} \frac{(-1)^{m}}{(2 m+1)^{1+\alpha}},
$$

where $D_{\alpha}=\sigma^{\alpha}$.

The maximal MFPT is assumed for $\alpha \simeq 1$. The behavior of the MFPT assumes a bell-shaped behavior around $\alpha=1$; it reflects the different interplay of the probability of finding long jumps versus a decreasing stability index $\alpha<2$, implying a decreasing noise intensity $\sigma^{\alpha}$. Put differently, the occurrence of long jumps beyond the finite interval is dominating the escape over a decreasing noise intensity for for $\alpha<1$.

The expected discrepancy between these analytical results and numerical estimation is due to assumed local boundary condition of vanishing probabilities $p(x=0)=$ $p(x=L)=0$, which are correct only for normal Brownian motion; i.e. $\alpha=2$. Indeed, our numerical result just coincides precisely at this very special value. In our simulation of the Langevin equation in (2) the whole exterior of the prescribed interval $\left(B_{1}=0, B_{2}=4\right)$ is absorbing throughout, while for the analytical calculations the flawed, point-like boundary conditions are assumed [22].

Clearly, the extension of the absorbing regime to the whole two semi-lines outside the confining interval yields the physically correct value that accounts for the escape of the particle from the interval via long jumps. The numerical results for $\alpha<2$ systematically exceed the theory-result of Ref. [22].

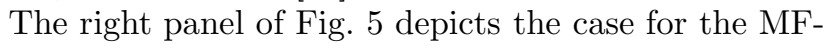
PTs with AR and RA boundaries, respectively. Due to symmetric chosen initial condition and the symmetric stochastic driving the results for AR and RA become identical.

\section{MFPT for asymmetric Lévy noise}

Asymmetric Lévy noise is characterized by a nonvanishing skewness parameter $\beta \neq 0$ (see the exemplary probability density functions in Fig. 6). Fig. 7 presents the results of MFPT evaluations for fully asymmetric Lévy stable noise driving with $|\beta|=1$. The left panel displays results for the Lévy-Brownian motion with $\beta=1$ within the AA boundaries setups. Due to the imposed symmetry of a starting point, the results coincide with those for the Lévy noise driving with $\beta=-1$. The right panel depicts the results for AR boundaries with $\beta= \pm 1$. As can be inferred by inspection of Fig. [6] due to the skewness character of the Lévy stable distribution, the 
results for AA boundaries with $\alpha<1$ and $\beta=1$ are the same as for $\mathrm{AR}$ boundaries with $\alpha<1$ and $\beta=-1$. The effect is caused by a visible shift of the probability mass apart from $x=0$ to the left (or to the right) for skewed distributions with $\alpha<1$. In contrast, these results differ for $\alpha>1$ where both boundary setups, i.e. AA and AR, lead to different values for MFPTs.
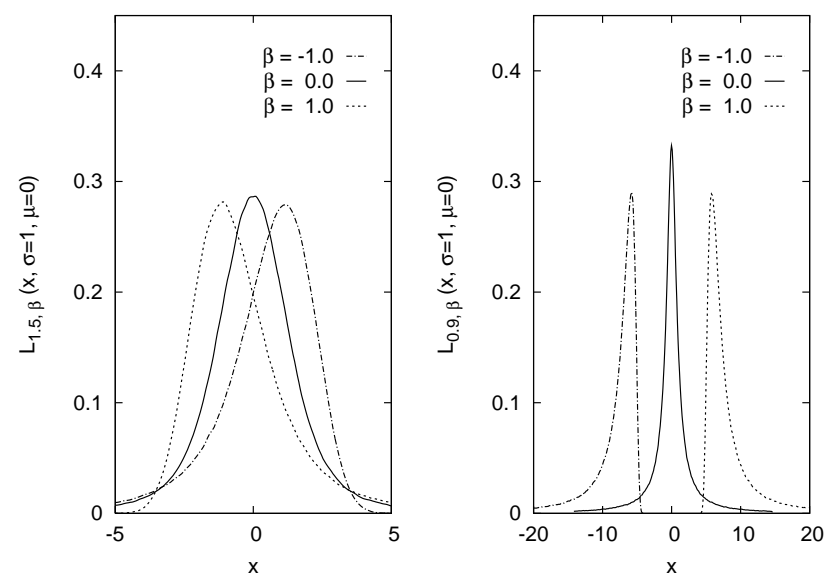

FIG. 6: Probability density functions for the $\alpha$-stable variables $x$ with $\alpha=1.5$ (left panel) and $\alpha=0.9$ (right panel). The symmetric case is for $\beta=0$, while $\beta= \pm 1$ corresponds to asymmetric densities. Note the differences in the positions of the maxima for $\alpha<1$ and $\alpha>1$. Most importantly, the support of the densities for the fully asymmetric cases with $\beta= \pm 1$ and $\alpha<1$ (right panel) is covering not the whole axis; it assumes only negative values for $\beta=-1$ and only positive values for $\beta=1$. This in turn causes the discontinuous behavior depicted with Fig. [7]
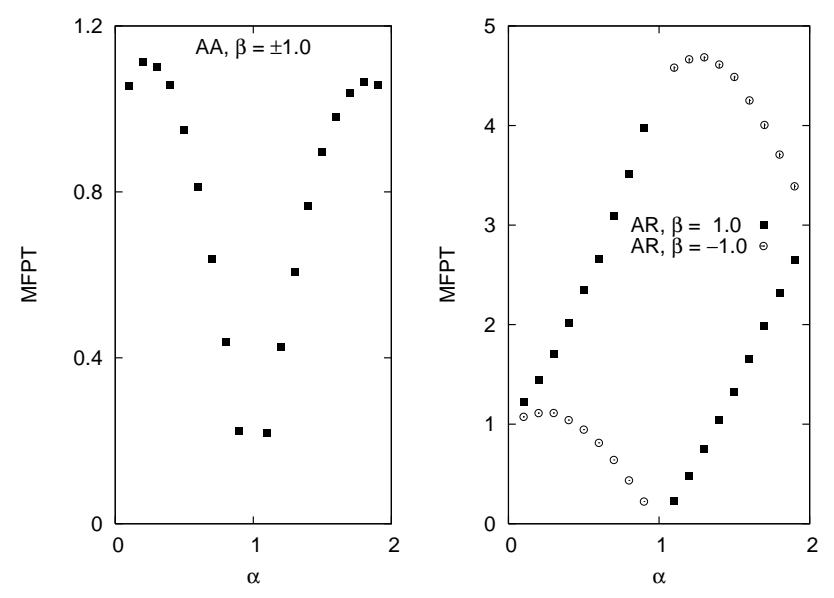

FIG. 7: MFPTs versus the stability index $\alpha$ for asymmetric Lévy noise for different boundary conditions: AA (left panel), AR (right panel). The simulations parameters are as in Fig. 5 Note that the results for the AR setting with $\beta=-1$ and $\alpha<1$ are the same as for the AA settings with $\beta \pm 1$ and $\alpha<1$.

As can be intuitively expected, the cumulative first passage time distributions (CDFs) of the first passage times as governed by Eq. (2) for the AA boundaries with a symmetric starting point, i.e. $x_{0}=L / 2$, are invariant under the transformation $\beta \rightarrow-\beta$. Therefore, the quantities derived from the numerically determined CDFs are symmetric around $\beta=0$, cf. the left panel of Fig. [ Furthermore, because of the chosen symmetric initial conditions the cumulative first passage time distributions evaluated for the RA boundaries setup can be constructed from the appropriate distribution for the AR case by exchanging $\beta$ with $-\beta$. It is caused by the fact that the asymmetry induced by a nonzero skewness parameter can be compensated by an exchange of boundaries, cf. left panel of Fig. 8 and Fig. [6.
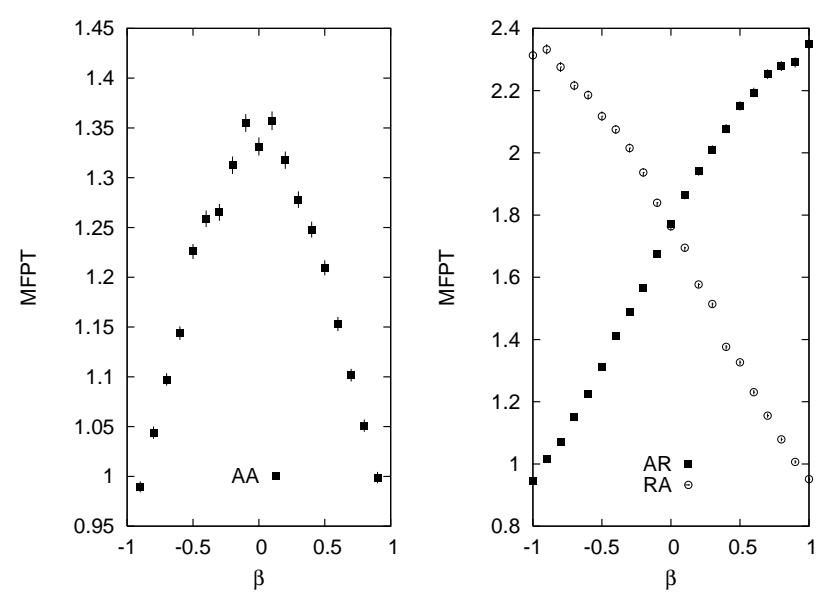

FIG. 8: MFPTs versus the skewness parameter $\beta$ for AA (left panel) and AR (right panel) boundaries for Lévy-Brownian motion with $\alpha=0.5$. The simulations parameters are like in Fig. 5

\section{First passage time statistics}

The simulation data for the mean first passage time yield as well the results for the cumulative first passage time distribution functions (CDFs). Typical such distributions are depicted in Fig. 9 for symmetric $\alpha$-stable noise and in Fig. 10 for asymmetric stable Lévy white noises with a stability index $\alpha=1.5$. With symmetric noise we observe that the escape, starting out at midpoint, is speeded up with two absorbing boundaries $\mathrm{AA}$ as compared to the situation with $\mathrm{AR}=\mathrm{RA}$. The reflecting boundary clearly slows down the ultimate escape from the finite interval.

For escape driven by asymmetric white Lévy noise, see Fig. 10] we note that for the case of two absorbing boundaries AA (left panel) the CDFs become identical for $\beta=-\beta$, given the midpoint starting value. We also can detect a more rapid saturation with $\beta \neq 0$ as compared to the fully symmetric situation with $\beta=0$; implying a somewhat faster escape scenario. This fact originates from the skewness in the distribution of jump 
values, implying a faster escape towards the corresponding absorbing boundary.

With an AR boundary setup the situation becomes more intricate. Now, depending on the choice of the asymmetry parameter the escape can be enhanced, reflecting the skewness of the corresponding stable distribution and the relative character of the boundary setup.
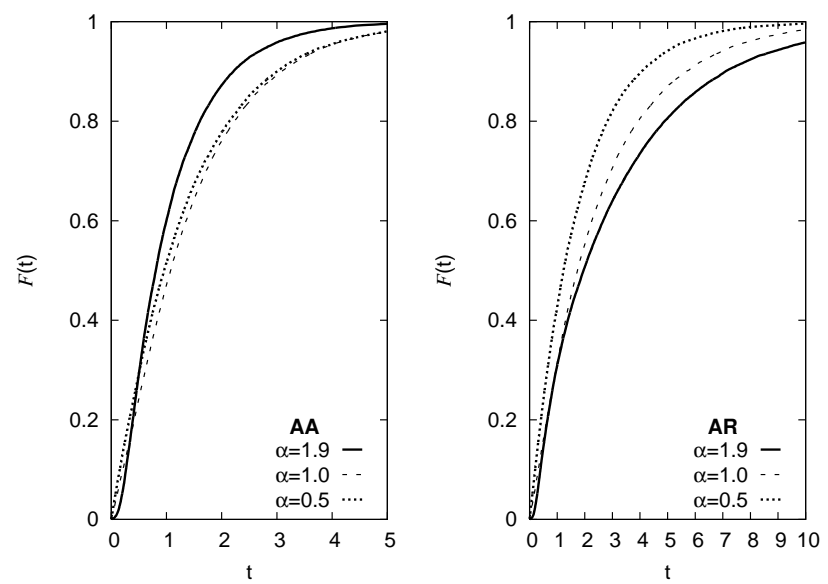

FIG. 9: Cumulative first passage time distribution function for symmetric Lévy-Brownian motion on a restricted interval, i.e. $\beta=0$, with $\alpha=1.9,1.0,0.5$ for AA (left panel) and AR (right panel) boundaries. The results have been simulated for $\sigma=\sqrt{2}, \Delta t=10^{-4}$ and have been averaged over $N=2 \times 10^{2}$ realizations. The boundaries $B_{1}, B_{2}$ are located at $x=0$ and $x=4$. The particle starts out at midpoint; i.e. $x_{0}=2$.
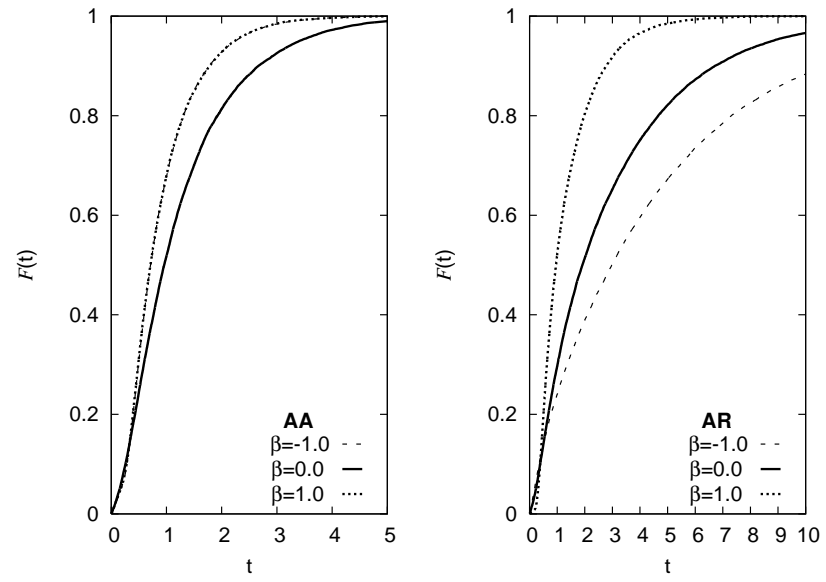

FIG. 10: Cumulative first passage time distribution function versus first passage time $t$ for Lévy noises with $\alpha=1.5$ and $\beta=-1.0,0.0,1.0$ for AA (left panel) and AR (right panel) boundaries. The simulations details are the same as in Fig. 5 Note that the results for the AA settings with $\beta= \pm 1$ overlap.

This complexity is elucidated further in panel 11] for the case of stable Lévy-Smirnoff noise where $\alpha=0.5, \beta=$ 1. The $\operatorname{CDF} \mathcal{F}(t)$ is depicted in Fig. 11] For $\alpha<1$ and $|\beta|=1$ Lévy stable distributions are totally skewed, taking on either only positive values $(\beta=1)$ or negative values only $\beta=-1$. Therefore, the results for $\alpha<1$ and $|\beta|=1$ for $\mathrm{AA}$ boundaries are the same as for $\mathrm{AR}$ $(\beta=-1)$ or $\mathrm{RA}(\beta=1)$ boundaries. This effect is clearly visible for the Lévy-Smirnoff case depicted in Fig. 11] Now, the results for AA and RA boundaries are the same, as expected. A similar behavior is observed in Fig. 7 above, where the values of the MFPTs for $\alpha<1$ for AA and AR boundaries agree.

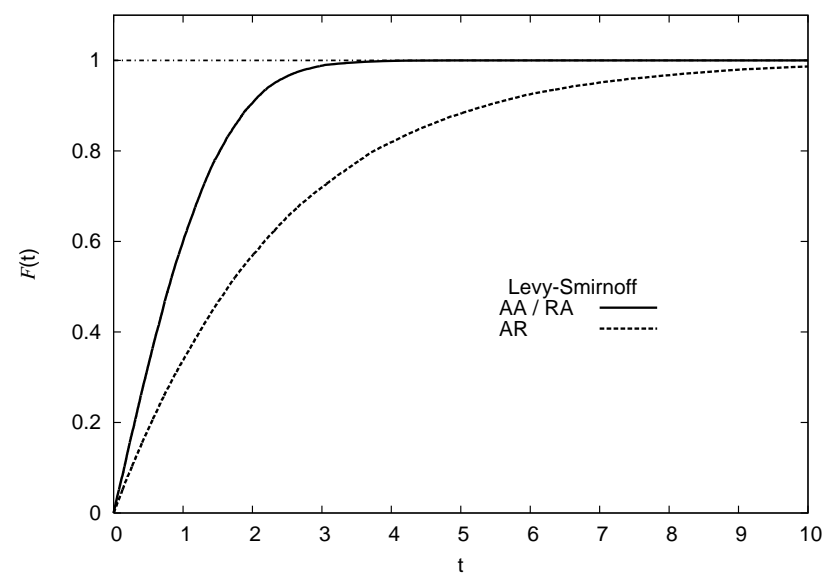

FIG. 11: The cumulative first passage time distribution $\mathcal{F}(t)$ versus first passage time $t$ for Lévy-Smirnoff driven LévyBrownian motion, i.e. $\alpha=0.5$ and $\beta=1$ and a scale parameter $\sigma=\sqrt{2}$. Because the Lévy-Smirnoff statistics assumes only positive values the distributions $\mathcal{F}(t)$ for AA and RA configurations become the same. The simulations parameters are as in Fig. 5

\section{Survival probability: comparison with Sparre-Andersen scaling}

The survival probability $\mathcal{S}(t)=1-\mathcal{F}(t)$ for symmetric Lévy-Brownian motion on the finite interval is displayed in Fig. 12 The motion on a confined support leads to an exponential decay of the survival probability with the steepness of the slope depending on the stability index $\alpha$. Notable differences can be observed for various boundary setups: For motion occurring between two absorbing boundaries, the survival probability decays faster than for the case when one of the boundaries is taken as reflecting. It is also useful to emphasize that deviations from the exponential behavior of the survival probability can be observed in systems subjected to both, dichotomic and Lévy stable noises [33, 34], respectively.

Finally, for the systems driven by Lévy stable noises we also tested the Sparre-Andersen scaling behavior on an infinite half line. According to the Sparre-Andersen theorem [13] for a free stochastic processes driven by symmetric white noises, the first passage time densities $\frac{d \mathcal{F}}{d t}$, process from the real half line asymptotically behave like $t^{-3 / 2}$. Consequently the survival probability, i.e. the probability of finding a particle starting its motion at 

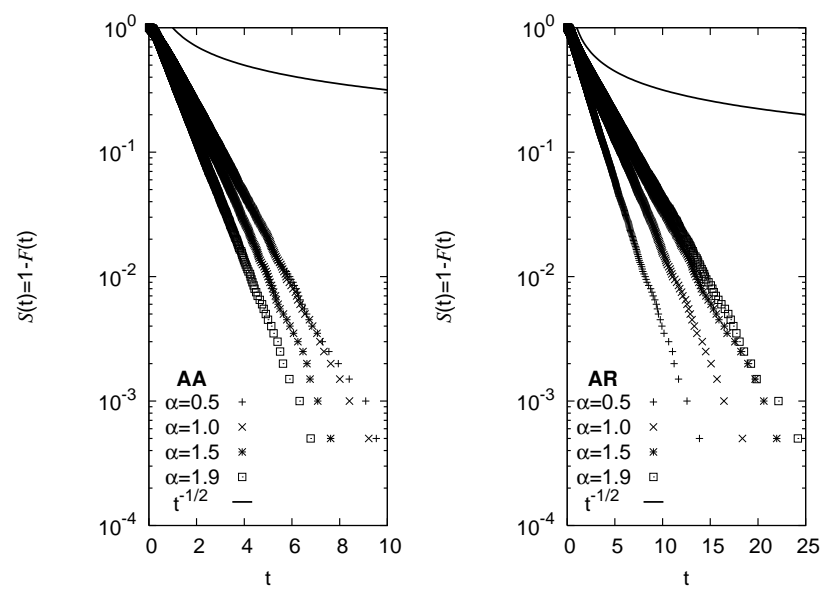

FIG. 12: Survival probability $S(t)=1-\mathcal{F}(t)$ for confined, symmetric Lévy-Brownian motion $\beta=0$ on the interval $B_{1}=0, B_{2}=4$ with absorbing half-line for $x<0$ and absorbing (or reflecting) half-line for $x>0$ for various values of the stability index $\alpha$ and midpoint initial conditions at $x_{0}=2$. The left panel depicts the results for AA boundary setups; the AR cases are displayed with the right panel. The solid line in both data sets represents the power law $t^{-1 / 2}$ which describes an asymptotic behavior foreseen by the Sparre-Andersen theorem [13] for diffusion on semi-infinite intervals.

$x_{0}>0$ in the real half line, scales like $t^{-1 / 2}$. In Fig. 13 the survival probability $S(t)=1-\mathcal{F}(t)$ is depicted for various stability indices $\alpha$ and various initial conditions $x_{0}$. It is clearly visible that the survival probability $S(t)$ behaves like a power law with the exponent $(-1 / 2)$, as predicted by the Sparre-Andersen theorem. For the testing of the Sparre-Andersen theorem the whole negative half line was assumed to be absorbing.

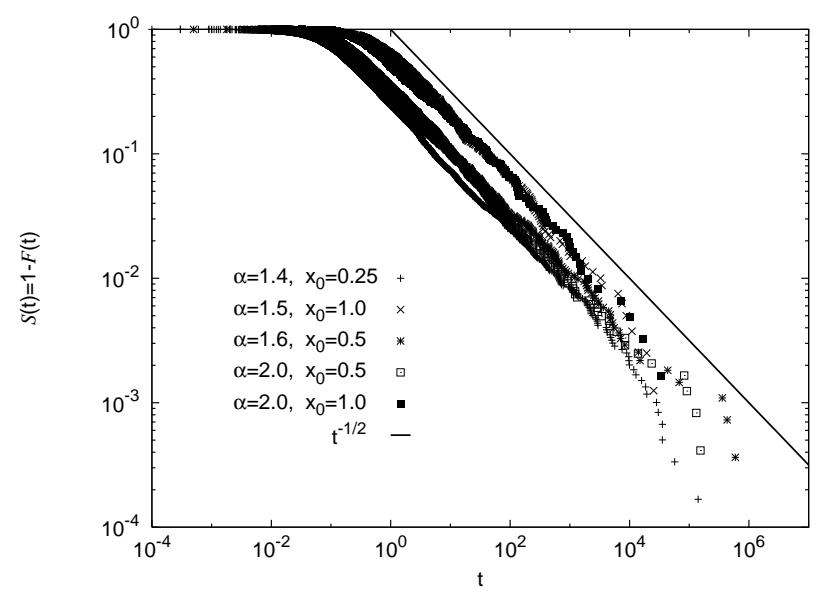

FIG. 13: Survival probability $S(t)=1-\mathcal{F}(t)$ for free Lévy flights on the half line with absorbing boundary for $x<0$ for various values of stability index $\alpha$ and various initial conditions $x_{0}$. Remaining simulation parameters like in Fig. 5 ] The survival probability nicely fits $t^{-1 / 2}$ slope predicted by Sparre-Andersen scaling.

\section{CONCLUSIONS}

With this work we investigated the problem of the mean first passage time and the first passage time statistics for Markovian, Lévy-Brownian motion proceeding on a finite interval. The intricate problem of setting up the proper boundary conditions for absorption and reflection are discussed with possible pitfalls being pointed out. In particular, it has been demonstrated by numerical studies that the use of the commonly known, local boundary condition of vanishing flux (in case of reflection) and vanishing probability (in case of absorbtion), valid for normal Brownian motion (i.e. $\alpha=2$ ) no longer apply for Lévy white noise. This is so, because the large, jump like excursions of Lévy flight increments causes noncontinuous, i.e. discontinuous sample trajectories. This in turn requires the use of nonlocal boundary conditions. It is presently not known how these nonlocal boundary conditions can be recast as in equivalent form as a modified, locally defined differential condition involving the statistical quantities of interest (the MFPT and the first passage time densities) at the location of the boundary alone [35, 36].

For symmetric, $\alpha$ stable Lévy white noise driven Brownian motion we find a bell-shaped, non-monotonic behavior of the MFPTs for absorbing-absorbing boundaries, with the maximum being assumed for $\alpha=1$. In contrast, for reflecting-absorbing boundaries we find a monotonic increase. With asymmetric Lévy white noise, i.e. with a non-vanishing skewness parameter $\beta \neq 0$ the MFPT results in an even more complex behavior. As a function of the stability index $\alpha$ the MFPT can exhibit a discontinuous behavior, see the right panel in Fig. 7

In addition we have studies also the statistics of the first passage times in terms of the cumulative first passage time distribution function (CDF) and the corresponding survival probability. In this context, we also belabor the role of a finite support and different boundary setups for Lévy white noise driven Brownian motion for the universal scaling law of Sparre-Andersen. While the restricted Lévy-Brownian motion exhibits an exponential behavior on finite intervals, the crossover to the universal $t^{-1 / 2}$ - power law, being valid on the half-line for all stability indexes is assumed for large intervals only.

\section{APPENDIX A: $\alpha$-STABLE RANDOM VARIABLES}

The random variables $\zeta$ corresponding to the characteristic functions (3) and (4) can be generated using the Janicki-Weron algorithm [16, 17]. For $\alpha \neq 1$, their representation reads

$$
\begin{aligned}
\zeta & =D_{\alpha, \beta, \sigma} \frac{\sin \left(\alpha\left(V+C_{\alpha, \beta}\right)\right)}{(\cos (V))^{\frac{1}{\alpha}}} \\
& \times\left[\frac{\cos \left(V-\alpha\left(V+C_{\alpha, \beta}\right)\right)}{W}\right]^{\frac{1-\alpha}{\alpha}},
\end{aligned}
$$


with the constants $C, D$ given by

$$
\begin{gathered}
C_{\alpha, \beta}=\frac{\arctan \left(\beta \tan \left(\frac{\pi \alpha}{2}\right)\right)}{\alpha}, \\
D_{\alpha, \beta, \sigma}=\sigma\left[\cos \left(\arctan \left(\beta \tan \left(\frac{\pi \alpha}{2}\right)\right)\right)\right]^{-\frac{1}{\alpha}} .
\end{gathered}
$$

For $\alpha=1$, the random variable $\zeta$ can be calculated from the formula

$$
\left.\zeta=\frac{2 \sigma}{\pi}\left[\left(\frac{\pi}{2}+\beta V\right) \tan (V)-\beta \ln \left(\frac{\frac{\pi}{2} W \cos (V)}{\frac{\pi}{2}+\beta V}\right)\right] \mathrm{A} 4\right)
$$

In the above equations $V$ and $W$ denote independent random variables; namely, $V$ is uniformly distributed in the interval $\left(-\frac{\pi}{2}, \frac{\pi}{2}\right)$ while $W$ is exponentially distributed with a unit mean [16]. The numerical integration scheme has been performed for $\mu=0$ with the increments of $\Delta x$ (see Eqs. (2) and (5)) sampled from the strictly stable distributions [19].

The analytical expressions for stable probability distributions $L_{\alpha, \beta}(\zeta ; \sigma, \mu)$ are known in few cases only: For $\alpha=0.5, \beta=1$ the resulting distribution is the LévySmirnoff one; i.e.,

$$
\begin{aligned}
L_{1 / 2,1}(\zeta ; \sigma, \mu) & =\left(\frac{\sigma}{2 \pi}\right)^{\frac{1}{2}}(\zeta-\mu)^{-\frac{3}{2}} \\
& \times \exp \left(-\frac{\sigma}{2(\zeta-\mu)}\right) .
\end{aligned}
$$

In contrast, for $\alpha=1, \beta=0$ one obtains the Cauchy distribution

$$
L_{1,0}(\zeta ; \sigma, \mu)=\frac{\sigma}{\pi} \frac{1}{(\zeta-\mu)^{2}+\sigma^{2}} .
$$

The familiar case with $\alpha=2$ with arbitrary $\beta$ yields the Gaussian PDF. The prominent characteristic feature of the distributions $L_{\alpha, \beta}(\zeta ; \sigma, \mu)$ is its existence of moments up to the order $\alpha$, i.e. the integral $\int_{-\infty}^{\infty} L_{\alpha, \beta}(\zeta ; \sigma, \mu) \zeta^{\alpha} d \zeta$ is finite. This statement results in the conclusion that the only stable distribution possessing a finite second moment is the Gaussian; for all other values of $\alpha$ the variance of a stable distribution diverges, and for $\alpha<1$ even the first moment does not exist.

\section{ACKNOWLEDGMENTS}

The Authors acknowledge the financial support from the Polish State Committee for Scientific Research (KBN) through the grants 1P03B06626 (2004-2005) and 2P03B08225 (2003-2006) and the ESF funds (E.G.N. and P.H.) via the STOCHDYN program. Additionally, BD acknowledges the financial support from the Foundation for Polish Science through the domestic grant for young scientists (2005). Computer simulations have been performed at the Academic Computer Center CYFRONET AGH, Kraków.
[1] M.F. Shlesinger, G.M. Zaslavsky, U. Frisch Eds., Lévy Flights and Related Topics in Physics (Proc. Nice, France; Springer Verlag, 1994).

[2] B.D. Hughes, Random Walks and Random Environments(Oxford Science, Oxford, 1995), Vol. I.

[3] R. Metzler and J. Klafter, Phys. Rep. 339, 1 (2000).

[4] P.M. Drysdale and P.A. Robinson, Phys. Rev. E 58, 5382 (1998).

[5] I.M. Sokolov, J. Mai, and A. Blumen, Phys. Rev. Lett 79, 857 (1997).

[6] P.D. Ditlevsen, Geophys. Res. Lett. 26, 1441 (1999); P.D. Ditlevsen, M.S. Kristensen and K.K. Andersen, J. Climate 18, 2594 (2005).

[7] P. Santini, Phys. Rev. E. 61, 93 (2000).

[8] D.R. Cox, H.D. Miller, The Theory of Stochastic Processes (Chapman and Hall, London, 1965).

[9] N.S. Goel and N. Richter-Dyn, Stochastic Models in Biology (Academic Press, New York, 1974).

[10] P. Hänggi, P. Talkner and M. Borkovec, Rev. Mod. Phys. 62, 251 (1990).

[11] A.V. Chechkin, R. Metzler, V.Y. Gonchar, J. Klafter, and L.V. Tanatarov, J. Phys. A. 36, L537 (2003).

[12] R. Metzler and J. Klafter, J. Phys. A 37, R161 (2004).

[13] E. Sparre Andersen, Math. Scand. 1, 263 (1953).

[14] B.J. West, V. Seshadri, Physica A 113, 203 (1982).

[15] M.E.J. Newman and G.T. Barkema, Monte Carlo Methods in Statistical Physics (Clarendon Press, Oxford,
1999).

[16] A. Weron and R. Weron, Lecture Notes in Physics 457, 379 (1995).

[17] R. Weron, Statist. Prob. Lett. 28, 165 (1996).

[18] P.D. Ditlevsen, Phys. Rev. E 60, 172 (1999).

[19] A. Janicki and A. Weron, Simulation and Chaotic Behavior of $\alpha$-Stable Stochastic Processes (Marcel Dekker, New York, 1994).

[20] A. Janicki, Numerical and Statistical Approximation of Stochastic Differential Equations with Non-Gaussian Measures (Hugo Steinhaus Centre for Stochastic Methods, Wrocław, 1996).

[21] B. Dybiec and E. Gudowska-Nowak, Phys. Rev. E 69, 016105 (2004).

[22] M. Gitterman, Phys. Rev. E 62, 6065 (2000).

[23] Note that in Eq. (39) of Ref. 22] the symbol $n$ should read $m$ and the sum should start out at $m=0$.

[24] M. Gitterman, Phys. Rev. E 69, 033102 (2004).

[25] G.M. Zaslavsky, Physica D 76, 110 (1994).

[26] H.C. Fogedby, Phys. Rev. E 58, 1690 (1998).

[27] V.V. Yanovsky, A.V. Chechkin, D. Schertzer, and A.V. Tur, Physica A 282, 13 (2000).

[28] I. Eliazar and J. Klafter, J. Stat. Phys. 111, 739 (2003).

[29] A. Dubkov and B. Spagnolo, Fluct. Noise Lett. 5, L267 (2005).

[30] S.V. Buldyrev et al., Physica A 302, 148 (2001).

[31] S.V. Buldyrev et al., Phys. Rev. E 64, 041108 (2001). 
[32] V. Balakrishnan, C. Van den Broeck, and P. Hänggi, Phys. Rev. A 38, 4213 (1988).

[33] B. Dybiec and E. Gudowska-Nowak, Fluct. and Noise Lett. 4, L273 (2004).

[34] B. Dybiec and E. Gudowska-Nowak, Proc SPIE 5467,
411 (2004).

[35] C. Van den Broeck and P. Hänggi, Phys. Rev. A 30, 2730 (1984).

[36] G.H. Weiss and A. Szabo, Physica A 119, 569 (1983). 